\title{
The Analysis of the Quenching Efficiency of Humic Acid Fluorescence by Cadmium and Copper Ions
}

\author{
Nikolay L. Lavrik, Nurullo U. Mulloev \\ Siberian Branch of the Russian Academy of Sciences, Voevodsky Institute of Chemical Kinetics and Combustion, \\ Novosibirsk, Russia \\ Email: lavrik@ns.kinetics.nsc.ru
}

Received 17 September 2014; revised 2 November 2014; accepted 18 November 2014

Copyright @ 2014 by authors and Scientific Research Publishing Inc.

This work is licensed under the Creative Commons Attribution International License (CC BY). http://creativecommons.org/licenses/by/4.0/

c) (i) Open Access

\begin{abstract}
In conditions of monochromatic excitation $\left(\lambda_{\text {exc }}=337.1 \mathrm{~nm}\right)$, the spectral dependence of the efficiency of fluorescence quenching $\alpha_{\lambda}$ of humic acids samples by $\mathrm{Cd}^{2+}$ and $\mathrm{Cu}^{2+}$ ions was studied. The difference of $\alpha_{\lambda}$ dependencies for these ions was established. In the spectral range 350 - 650 $\mathrm{nm}$, changes of the $\alpha_{\lambda}$ for $\mathrm{Cd}^{2+}$ ion are markedly different from the magnitude for changes $\alpha_{\lambda}$, which occur for ion $\mathrm{Cu}^{2+}$. The interpretation of mismatch dependences obtained $\alpha_{\lambda}$ ions $\mathrm{Cd}^{2+}$ and $\mathrm{Cu}^{2+}$ is carried out within the concept of the availability of different sites, due to the difference in ionic radii $\left(0.108 \mathrm{~nm}\right.$ and $0.08 \mathrm{~nm}$ for $\mathrm{Cd}^{2+}$ and $\mathrm{Cu}^{2+}$, respectively).
\end{abstract}

\section{Keywords}

Structure of Humic Acids, Spectral Dependence of Fluorescence Quenching, $\mathrm{Cd}^{2+}$ and $\mathrm{Cu}^{2+} \mathrm{Ions}^{2}$

\section{Introduction}

Humic acids (HAs), regardless of their origin, are high nitrogen-containing organic acid molecules that contain aromatic groups. The analysis of the results obtained by a number of direct and indirect methods is allowed to create an overall model of the structure of macromolecules of HA: its core comprises aromatic carbon skeleton and the periphery contains polysaccharide-polypeptide chains [1]. The studies of the conformational properties of HAs are highly relevant because HAs play a crucial role in the formation of agriculturally important soil structure and the structure of HA largely determines its physical and chemical properties. In addition, the relevance of studying of the conformational properties of HA is due to their ability to reduce the effect of toxicants as 
the latter bind into HA complexes.

A characteristic structural feature of macromolecules of HAs is their variability and polydispersity of their elemental and molecular composition [1]. To assess the degree of polydispersity of HAs the numerous physicalchemical methods were used [1]-[5], and in particular, fluorescent approaches [6]-[13]. Earlier [12], we proposed an original method for assessing the degree of polydispersity of the sample HA. This method is based on the excitation of the fluorescence by monochromatic illumination. This technique was able to detect the spectral dependence of the fluorescence quenching efficiency. The possibility of such approach has been demonstrated by fluorescence quenching of the standard sample $\mathrm{HA}$ by $\mathrm{Cu}^{2+}$ ion. In this experiment, monotonic spectral dependence of the quenching constants was not received and results in the conclusion were made available at least three spectral components.

The aim of this work was to compare the spectral dependence of the efficiency of fluorescence quenching the $\mathrm{HA}$ samples by $\mathrm{Cd}^{2+}$ and $\mathrm{Cu}^{2+}$ ions. These ions have different radii: $0.108 \mathrm{~nm}$ and $0.08 \mathrm{~nm}$ for $\mathrm{Cd}^{2+}$ and $\mathrm{Cu}^{2+}$, respectively [14]. In this regard, it can be expected that their interaction with sites containing fluorophores will be different. This difference may result from the differences in spatial accessibility of the quencher to the site containing the fluorophores (at the same value of Van der Vaals interaction) or from the differences in magnitude of Van der Vaals interaction (at the same available space). As a result of the experiments, it was found that the spectral dependence of the fluorescence quenching efficiency of $\mathrm{Cd}^{2+}$ ion, $\mathrm{HA}$, undergoes larger changes than the spectral dependence of the fluorescence quenching efficiency of $\mathrm{HA}$ by ion $\mathrm{Cu}^{2+}$.

\section{Materials and Methods}

\subsection{Materials}

As a sample, HA the standard preparation (Humic Asid Standard IHSS Elliot soil 1S102H) was used. We used alkaline solutions ( $\mathrm{NaOH}$, "Fluka"). It was used as the quencher cadmium nitrate $\mathrm{Cd}\left(\mathrm{NO}_{3}\right)_{2} \cdot 5 \mathrm{H}_{2} \mathrm{O}$ and copper sulfate $\mathrm{CuSO}_{4} \cdot 5 \mathrm{H}_{2} \mathrm{O}$ ("SCHDA" without further purification) were used in the experiments.

\subsection{Preparation of HA Solutions}

Stock solutions were prepared by dissolving HA $2 \mathrm{mg}$ of sample in $20 \mathrm{ml}$ of alkali solution. Solutions of lower concentrations were prepared by diluting this solution. The shaking of the initial solutions was performed for 60 minutes at room temperature followed by filtration. Measurement of the absorption spectra of the filtered and not filtered source HA solutions have shown their coincidence. Fluorescence spectra were recorded for 24 hours after the preparation of solutions.

Solutions of sulfuric and nitric acids were prepared using deionized water. When preparing solutions the scales "Sartorius" was used. The $\mathrm{pH}$ was $\sim 13$. $\mathrm{pH}$ values were measured with a $\mathrm{pH}$ meter "Redelkis". All experiments were performed at $(22 \pm 1)^{\circ} \mathrm{C}$.

\subsection{Fluorescence Equipment}

The absorption spectra were obtained on a Hewlett Packard 6041 spectrophotometer. Quartz cuvettes with optical path length of $1 \mathrm{~cm}$ were used. Fluorescence spectra were obtained $\mathrm{N}_{2}$-pulsed laser fluorometer $\left(\lambda_{\text {exc }}=337.1\right.$ $\mathrm{nm}$, illumination pulse frequency was $25 \mathrm{~Hz}$, the average power of $80 \mathrm{~mW}$ ) [12] [13]. The laser beam passed through the cell parallel to the top of the entrance slit of a fluorometer. The diameter of the laser beam was 3 $\mathrm{mm}$. Distance from the center of the laser beam to the wall of the working of the cell through which the fluorescence was recorded was $0.5 \mathrm{~cm}$. Cuvette was filled up with a solution to avoid formation of meniscus. Stability control of the intensity of the exciting light was conducted by periodic removal of the external standard (the intensity of Raman lines $\mathrm{OH}$ vibration of pure water).

\subsection{Processing of the Experimental Data}

Integral efficiency of fluorescence quenching was determined by the value of Stern-Volmer quenching constants $K_{\mathrm{sv}}$ [15] from the relation

$$
I_{0} / I=1+K_{\mathrm{SV}}\left[\mathrm{Me}^{2+}\right]
$$


In (1), $I_{0}$ and $I$-the corrected integral fluorescence intensity with $\mathrm{HA}\left[\mathrm{Me}^{2+}\right]=0$ and $\left[\mathrm{Me}^{2+}\right] \neq 0$, respectively ( $\mathrm{Me}^{2+}$ — cadmium or copper ion). The magnitude of the corrected integrated intensity $I$ determined from the equation (2)

$$
I=\left(1-10_{337}^{-\mathrm{OD}}\right)^{-1} \times \int\left(I_{\text {exp }, \text { solution }}(\lambda)-I_{\text {exp,solvent }}(\lambda)\right) \times 10^{\mathrm{ODem}, \lambda} \mathrm{d} \lambda
$$

In (2), $I_{\text {exp,solution }}(\lambda)$ and $I_{\text {exp,solvent }}(\lambda)$ experimentally observed fluorescence intensity of solutions and solvent by fluorescence wavelength $\lambda$ respectively. $\mathrm{OD}_{337}$ optical density at $\lambda=337.1 \mathrm{~nm} . \mathrm{OD}_{\mathrm{em}, \lambda}$-optical density at the wavelength of fluorescence $\lambda$ at optical path length of $0.5 \mathrm{~cm}$ in the spectral range $350-650$ $\mathrm{nm}$. Factors $\left(1-10_{337}^{-\mathrm{OD}}\right)^{-1}$ and $10^{\mathrm{ODem}, \lambda}$ are corrective amendments on the inner filter effect in the excitation and emission of fluorescence, respectively. Integration of the modified loop fluorescence spectrum was conducted in the spectral range from 350 to $650 \mathrm{~nm}$ (the values of the lower and upper limit for $\int$ sign).

Typically, the efficiency of fluorescence quenching at a specific wavelength $\lambda$ quantitatively determined by the rate constants of quenching Stern-Volmer $K_{\mathrm{sV}}^{\lambda}$ from the relation [15]

$$
I_{o \lambda} / I_{\lambda}=1+K_{\mathrm{SV}}^{\lambda}\left[\mathrm{Me}^{2+}\right]
$$

In (3), $I_{o \lambda}$ and $I_{\lambda}-\mathrm{HA}$ fluorescence intensity wavelength at $\lambda$ for $\left[\mathrm{Me}^{2+}\right]=0$ and $\neq 0$, respectively. $I_{\lambda}$ value was determined by the formula

$$
I_{1}=\left(I_{\text {exp,solution }}(\lambda)-I_{\text {exp }, \text { solvent }}(\lambda)\right) \times\left(1-10_{337}^{-\mathrm{OD}}\right)^{-1} \times 10^{\mathrm{ODem}, \lambda}
$$

However, in [13] it was shown that the magnitude $K_{\mathrm{sv}}^{\lambda}$ cannot always be obtained from the experimental data due to the fact that in conventional rectifiability anamorphoses Stern-Volmer may be missing. In this regard, in [13] to estimate the spectral efficiency of quenching was proposed to use a parameter

$$
\alpha_{\lambda}=I_{\text {o入 }} / I_{\lambda}
$$

Such a definition of quenching efficiency means that if $\alpha_{\lambda}>1$ holds fluorescence quenching, while $\alpha_{\lambda}<1$ the fluorescence intensity increases. In our work for the analysis of spectral efficiency of HA fluorescence quenching we also use this option. $\alpha_{\lambda}$ parameter characterizes the change in shape of the contour fluorescence upon addition of quencher and is a universal, i.e. it can be used for analysis of the spectra in all experiments. It is easy to see that, since $K_{\mathrm{SV}}^{\lambda}=\left(\alpha_{\lambda}-1\right) /\left[\mathrm{Me}^{2+}\right]$ (from (3)) and because any manifestation of non-monotony in the spectral dependence $K_{\mathrm{sV}}^{\lambda}$ manifest in the spectral dependence of the parameter $\alpha_{\lambda}$. An additional advantage of the parameter $\alpha_{\lambda}$ is that the analysis of spectral dependence of the quenching efficiency cannot ignore the correction factor $\left(1-10_{337}^{- \text {oD }}\right)^{-1}$ because calculation of the quantum yield is not required. Nevertheless, the determination of the $K_{\mathrm{sV}}$ (not $K_{\mathrm{SV}}^{\lambda}$ ) is crucial because it can help to evaluate the rate constant for quenching fluorescence $K_{q}$, which allows to draw conclusions about static or diffusion mechanism of fluorescence quenching [15].

In addition, the analysis of experimental data, we used the $\beta_{\lambda}$ parameter-the ratio of the $\alpha_{\lambda}$ values for $\mathrm{Cu}^{2+}$ and $\mathrm{Cd}^{2+}$ ions, respectively:

$$
\beta_{\lambda}=\alpha_{\lambda}^{\mathrm{Cu}^{2+}} / \alpha_{\lambda}^{\mathrm{Cd}^{2+}}
$$

From the definition of this value, it implies that for $\beta_{\lambda}>1$ copper ion more effectively quenches the fluorescence than cadmium ion, while $\beta_{\lambda}<1$ less efficiently.

Fluorescence quenching rate constant $K_{q}$ [15] evaluated the relation

$$
K_{q}=K_{\mathrm{sv}} / \tau_{0}
$$

In (7), $\tau_{0}$-fluorescence lifetime of the HA macromolecule in the absence of quencher. The $\tau_{0}$ values of HA samples ( $\sim 3$ ns) was taken from [10].

\section{Results}

The observed absorption spectra of these HA solutions were similar to obtained earlier in [12]. The execution of 
the Bouguer-Lambert-Beer law was satisfactory (data not shown).

Figure 1(a) and Figure 1(b) show the experimentally observed fluorescence spectra for the systems studied. In the inset, the Stern-Volmer dependences were showed, as amended by (2). It is visible good rectifiable. Evaluation of the constants $K_{\mathrm{sV}}$ gives values $(2560 \pm 250) \mathrm{M}^{-1}$ and $(4300 \pm 250) \mathrm{M}^{-1}$ for quenching ions $\mathrm{Cd}^{2+}$ and $\mathrm{Cu}^{2+}$, respectively. Quenching rate constants $K_{q}$ were $8.3 \times 10^{11} \mathrm{M}^{-1} \mathrm{~s}^{-1}$ and $1.4 \times 10^{12} \mathrm{M}^{-1} \mathrm{~s}^{-1}$ for quenching ions $\mathrm{Cd}^{2+}$ and $\mathrm{Cu}^{2+}$, respectively. These values exceed the diffusion constants, which for aqueous solutions should be around $10^{10} \mathrm{M}^{-1} \mathrm{~s}^{-1}$ [15]. Exceeding the observed quenching rate constants of diffusion values indicates the presence of static quenching, i.e. in the studied solutions the complex formation between HA macromolecules and metal ion takes place in the dark.
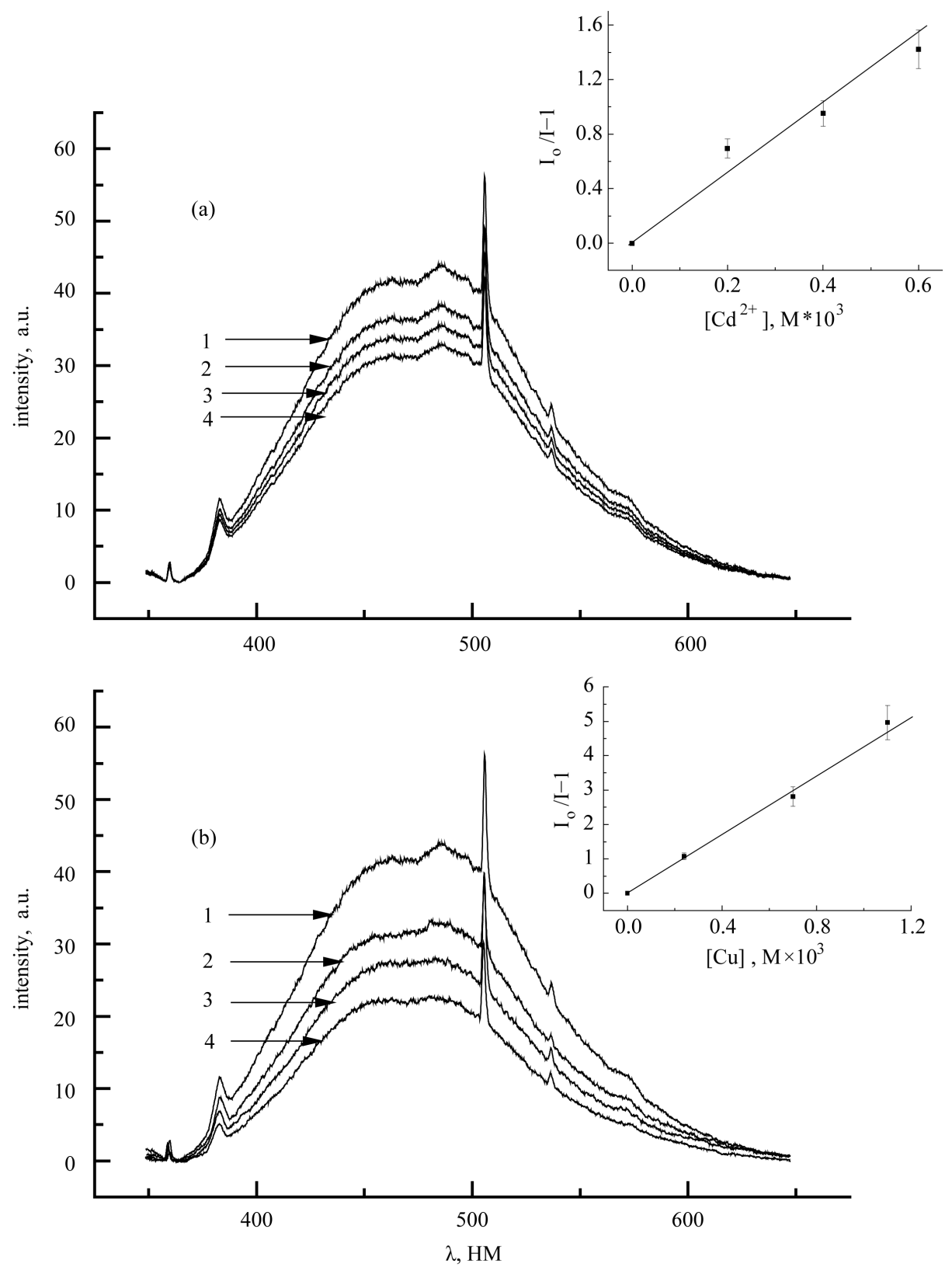

Figure 1. The experimentally observed fluorescence spectra of HA. $\left[\mathrm{Cd}^{2+}\right]=0(1) ; 2.5 \times 10^{-4} \mathrm{M}$ (2); $5 \times 10^{-4} \mathrm{M}(3) ; 1.1 \times 10^{-3} \mathrm{M}$ (4)-a; $\left[\mathrm{Cu}^{2+}\right]=0$ (1); $1.25 \times 10^{-3} \mathrm{M}(2) ; 2.5 \times 10^{-3} \mathrm{M}$ (3); $3.75 \times$ $10^{-3} \mathrm{M}$ (4)-b. The insets: the Stern-Volmer dependences constructed according to the Equation (2). 
Figure 2(a) shows the spectral dependence of $\alpha_{\lambda}$ for fluorescence quenching $\mathrm{HA}$ by ions $\mathrm{Cd}^{2+}(1)$ and $\mathrm{Cu}^{2+}$ (2). As can be seen from the figure, there is a noticeable difference in the spectral dependence of $\alpha_{\lambda}$ : in the spectral range $400<\lambda<600 \mathrm{~nm}$ cadmium ion quenches the fluorescence is weaker than the copper ion ( $\alpha_{\lambda}$ values for $\mathrm{Cd}^{2+}$ ion is less than for $\mathrm{Cu}^{2+}$ ion). This means that in the macromolecule IHSS the sites contained identical fluorophores interact weakly with the cadmium ion than with the copper ion. Noteworthy is also the fact that the observed value $\alpha_{\lambda}<1$ for cadmium ion at $\lambda>600 \mathrm{~nm}$, which means the increase of fluorescence intensity.

From of the data of Figure 2(a) the difference in the magnitude of changes in the efficiency of fluorescence quenching $\Delta \alpha_{\lambda}\left(\mathrm{Me}^{2+}\right)$ of copper and cadmium ions in the spectral range of $400-600 \mathrm{~nm}$ is also very noticeable:

$$
\Delta \alpha_{\lambda}\left(\mathrm{Me}^{2+}\right)=\alpha_{400}\left(\mathrm{Me}^{2+}\right)-\alpha_{600}\left(\mathrm{Me}^{2+}\right)
$$

For cadmium ion value $\Delta \alpha_{\lambda}\left(\mathrm{Cd}^{2+}\right)=0.69$ but for the copper ion $\Delta \alpha_{\lambda}\left(\mathrm{Cu}^{2+}\right)=0.308$. Thus, the difference of values is more than 2 times. This means that the interaction of cadmium ions with sites of HA macromolecule undergoes larger changes than the interaction of copper ions with the same sites. Thus, the availability of sites for HA fluorescence quenching for cadmium ions varies more than for copper ions.

In Figure 2(b), the spectral dependence of the $\beta_{\lambda}$ values is shown. In this case, the data clearly show that the dependence of the $\beta_{\lambda}$ on $\lambda$ is a function. The value increases with the increasing wavelength of HA fluorescence spectra. The form of the spectral dependence of $\beta_{\lambda}$ shows that upon increase of the "red" region of the spectrum fluorescence of the HA chromophore, the quenching efficiency of copper ion becomes higher.
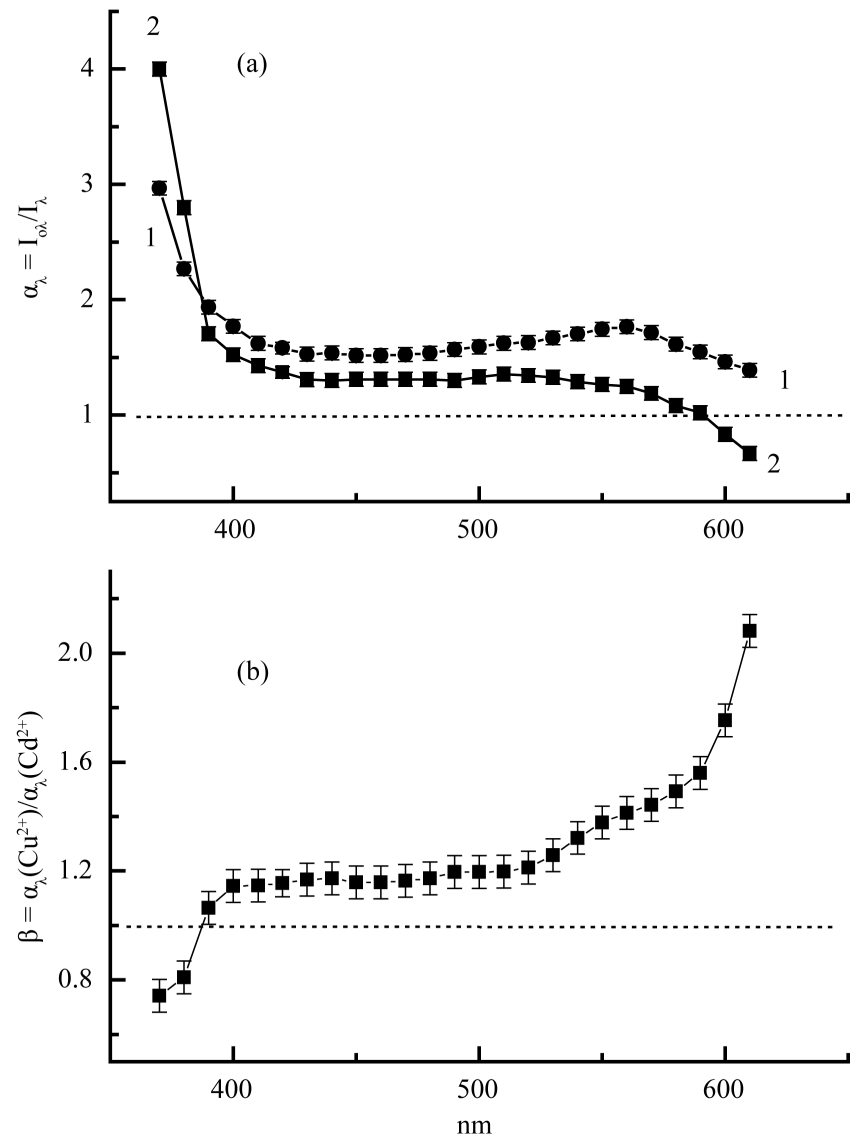

Figure 2. Spectral dependence of the $\alpha_{\lambda}$ parameter. (a) For copper ion-1; for cadmium ion-2. (b) Spectral dependence of the $\beta_{\lambda}$ parameter. 


\section{Discussion}

We obtained the following facts: First, in the spectral range 400 - $600 \mathrm{~nm}$ cadmium ion quenches the fluorescence weaker than the copper ion; second, in the spectral range 400 - $600 \mathrm{~nm}$ for the cadmium ion value $\Delta \alpha_{\lambda}$ is greater than for the copper ion; third, in the "blue" part of the spectrum of HA fluorescence the effective quenching by ions of both metals takes place; fourth; HA fluorescence quenching increases at $\lambda>600 \mathrm{~nm}$ upon adding cadmium ion; fifth, continuous growth values $\beta_{\lambda}$ with increasing $\lambda$ was observed.

1) The decrease of the effective quenching of cadmium ion can be explained by the low access to HA fluorophores by this ion due to its larger size compared to copper ion and, consequently, by the lower efficiency of forming the complex.

2) For all sites of HA with fluorophores emitting light in the range of $400-600 \mathrm{~nm}$, the availability of the copper ion is the same, while for the cadmium ion takes place a limit of availability.

3) Based on the proposed model, the effective quenching of ions of both metals in the "blue" part of the spectrum should be attributed to the fact that the chromophores emitting "blue" fluorescence are most accessible (not screened) and located, respectively, on the periphery of the HA macromolecule structure.

4) The increase of fluorescence intensity upon addition of cadmium ion $(\lambda>600 \mathrm{~nm})$ can be interpreted by the influence of the metal ion complex formation with the macromolecule HA and structure of the macro-molecule is changed partially. This result is a site in which the fluorophore undergoes complexation, which, in turn, increases its intensity. The increase in the fluorescence intensity upon the formation of complexes has been observed previously [15].

5) Increasing quantity of $\beta_{\lambda}$ with growing of $\lambda$ can be associated with the fact that binding of copper ions with specific sites of HA macromolecule takes place more effectively than cadmium ion in the case of sites having "red" fluorophores. Hence, the availability of sites having "red" fluorophores is higher for the smaller radius ion. This conclusion suggests that the "red" fluorophores have limited availability while availability of fluorophores causing "blue" region of the fluorescence spectrum of HA is much higher. In connection with this, the conservative assumption can be made that the "red" part of the fluorescence spectrum of HA contributes to the fluorescence of fluorophores located at the core sites of HA.

It is not difficult to see that the above explanation of the spectral dependences of $\alpha_{\lambda}$ is based on the assumption that the quenching efficiency depends only on the availability of fluorophores in HA structure. (The "chemical" factor was considered constant, e.g., the Coulomb interaction between the ions of copper and cadmium, with the same accessibility site is different due to different distances between the centers of interaction.). Currently, however, to give preference to the structural and chemical nature of the origin of the spectral dependence of quenching efficiency is not possible at this stage of knowledge.

\section{References}

[1] Stevenson, F.J. (1994) Humus Chemistry. Genesis, Composition, Reactions. 2nd Edition, John Wiley and Sons, Inc., New York.

[2] Visser, S.A. (1964) A Physico-Chemical Study of the Properties of Humic Acids and Their Changes During Humification. Journal of Soil Science, 15, 202-210. http://dx.doi.org/10.1111/j.1365-2389.1964.tb02219.x

[3] Ghosh, K. and Mukherjee, S.K. (1971) Hymatomelanic Acids as Polyelectrolytes. I. Viscometric and Osmometric Studies. Journal of Applied Polymer Science, 15, 2073-2081. http://dx.doi.org/10.1002/app.1971.070150902

[4] Khan, S.U. (1971) Distribution and Characteristics of Organic Matter in Humic Acids. Soil Science, 112, $410-416$.

[5] Wershaw, R.L., Burcar, P.J., Sutula, C.L. and Wiginton, B.J. (1967) Pyrolysis of Humic and Fulvic Acids. Science, 157, 1429-1435. http://dx.doi.org/10.1126/science.157.3795.1429

[6] Senesi, N., Miano, T.M., Provinzano, M.R. and Brunetti, G. (1991) Characterization, Differentiation and Classification of Humic Substances by Fluorescence Spectroscopy. Soil Science, 152, 259-271. http://dx.doi.org/10.1097/00010694-199110000-00004

[7] Zsolnau, A., Baigar, E. and Jimenez, M. (1998) Differentiating with Fluorescence Spectroscopy the Sources of Dissolved Organic Matter in Soils Subjected to Drying. Chemosphere, 38, 45-52. http://dx.doi.org/10.1016/S0045-6535(98)00166-0

[8] Lavrik, N.L. (2003) The Studying of Polydispersity Properties of Humic Asid by Help of Luminescence Spectroscopy. Chemistry for Sustainable Development, 11, 751-755.

[9] Lavrik, N.L. and Mulloev, N.U. (2006) The Influence of Humic Acid Concentration in Aqueous Solutions on the 
Structure of Their Macromolecules. Chemistry for Sustainable Development, 14, 379-390.

[10] Boyle, E.S., Guerriero, N., Thiallet, A., Veccino, R. and Blough, N.V. (2009) Optical Properties of Humic Substances and CDOM: Relation to Structure. Environment Science and Technology, 43, 2262-2268. http://dx.doi.org/10.1021/es803264g

[11] Lavrik, N.L. and Mulloev, N.U. (2010) Study of the Influence of Association on the Structure of Humic Acids by Fluorescence Quenching. Journal of Applied Spectroscopy, 77, 627-632. http://dx.doi.org/10.1007/s10812-010-9372-y

[12] Lavrik, N.L. and Mulloev, N.U. (2011) The Method for Studying Humic Acid Polydispersion by Fluorescence Quenching by $\mathrm{Cu}^{2+}$ Ions. Journal of Applied Spectroscopy, 78, 757-763. http://dx.doi.org/10.1007/s10812-011-9521-y

[13] Lavrik, N.L. and Mulloev, N.U. (2012) Concenration Dependence of Fluorescent Dependence of Humic Acids. Optika Atmospheri i Okeana, 25, 833-839 (Russia).

[14] Ravdel, A.A. and Ponomareva, A.M. (1983) Shot Reference of Physico-Chemical Values. 8th Edition, Chemistry, Moscow (Russia).

[15] Lakovicz, J. (2010) Principles of Fluorescence Spectroscopy. 3rd Edition, Springer, Berlin. 
Scientific Research Publishing (SCIRP) is one of the largest Open Access journal publishers. It is currently publishing more than 200 open access, online, peer-reviewed journals covering a wide range of academic disciplines. SCIRP serves the worldwide academic communities and contributes to the progress and application of science with its publication.

Other selected journals from SCIRP are listed as below. Submit your manuscript to us via either submit@scirp.org or Online Submission Portal.
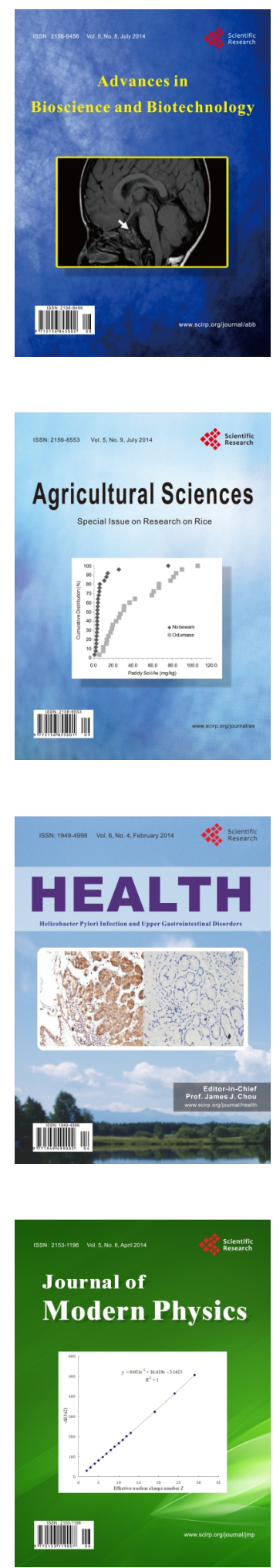
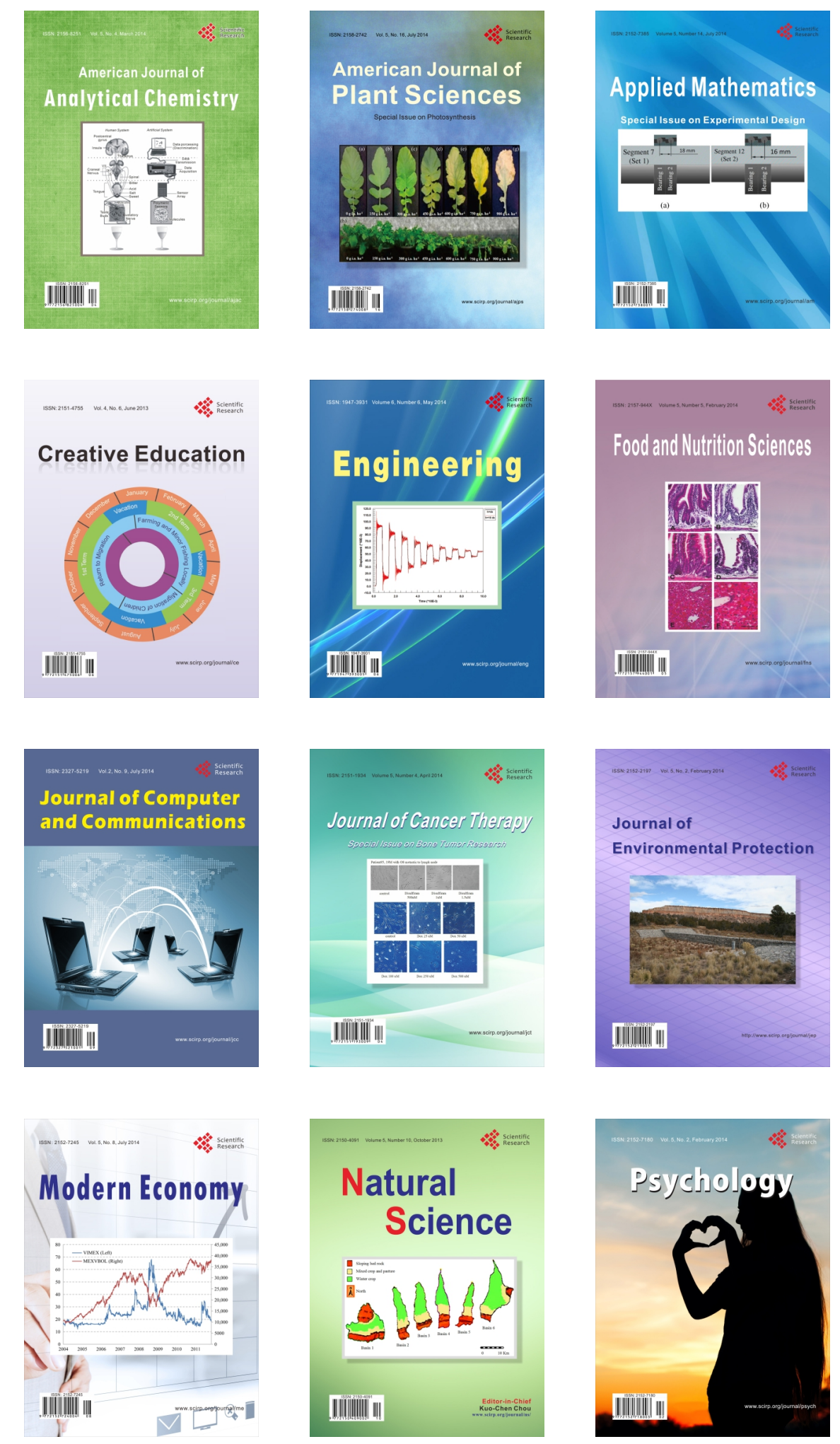